\title{
LA-UR-18-23318
}

Approved for public release; distribution is unlimited.

Title: MPA Materials Physics and Applications

Author(s): $\quad$ Kippen, Karen Elizabeth

Intended for: Brochure

Web

Issued:

2018-04-18 
Disclaimer:

Los Alamos National Laboratory, an affirmative action/equal opportunity employer, is operated by the Los Alamos National Security, LLC for the National Nuclear Security Administration of the U.S. Department of Energy under contract DE-AC52-06NA25396. By approving this article, the publisher recognizes that the U.S. Government retains nonexclusive, royalty-free license to publish or reproduce the published form of this contribution, or to allow others to do so, for U.S. Government purposes. Los Alamos National Laboratory requests that the publisher identify this article as work performed under the auspices of the U.S. Department of Energy. Los Alamos National Laboratory strongly supports academic freedom and a researcher's right to publish; as an institution, however, the Laboratory does not endorse the viewpoint of a publication or guarantee its technical correctness. 


\section{MPA Materials Physics and Applications}

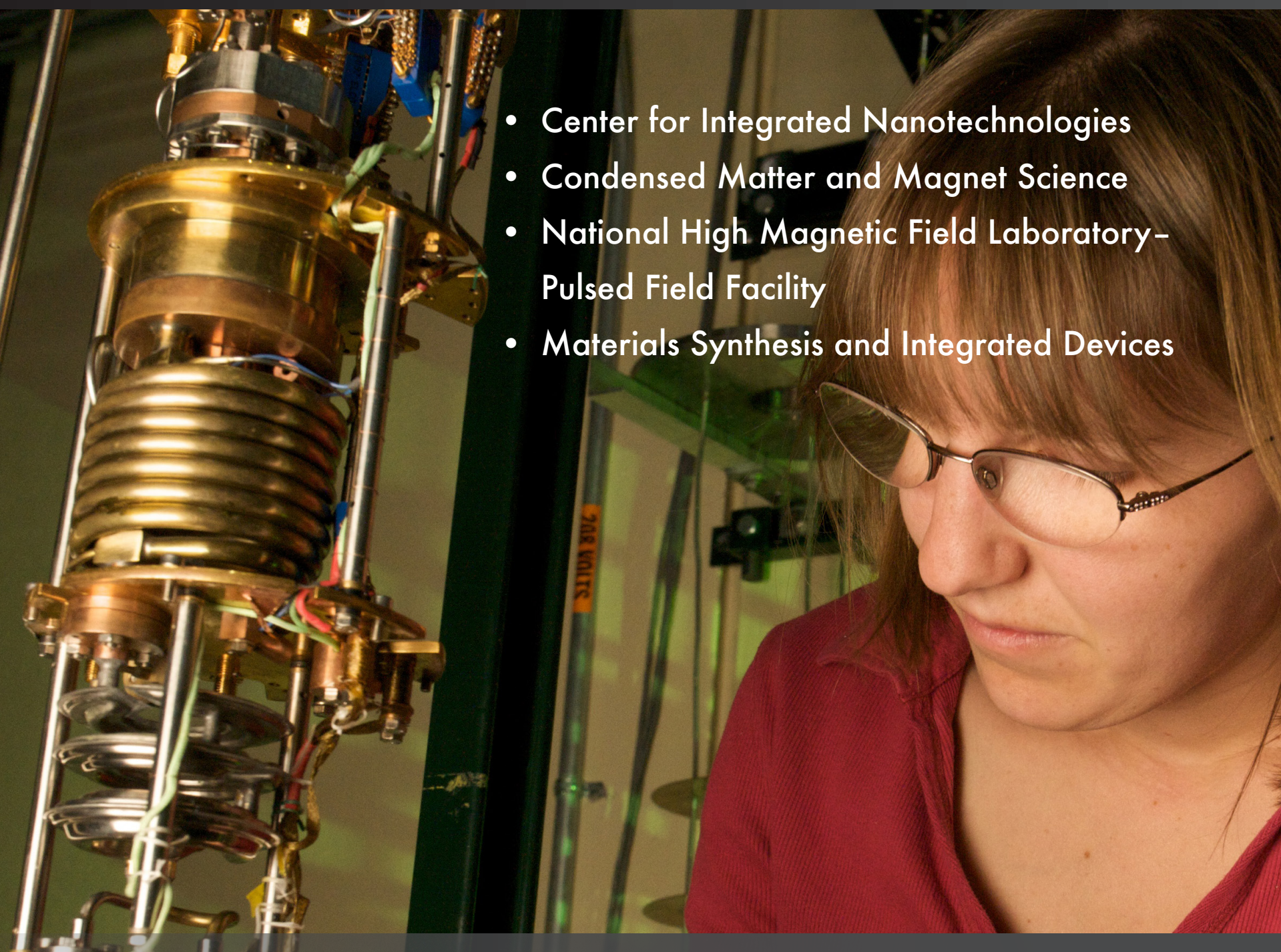

Science enabling the development of new technologies that

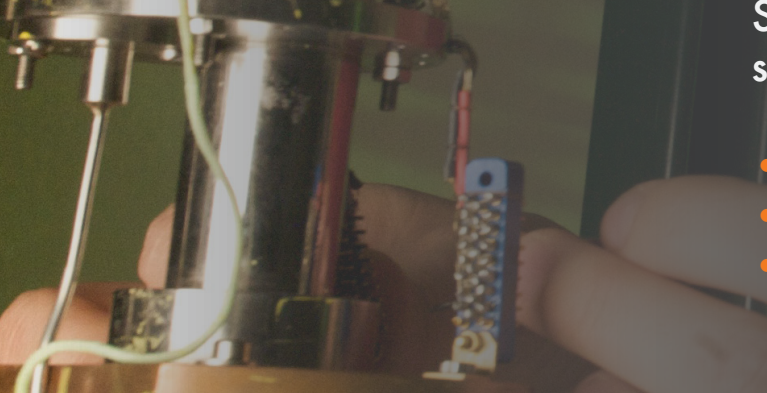

Tanja Pietraß, Division Leader | Rick Martineau, Deputy Division Leader Mail Stop F612 | Los Alamos National Laboratory | Los Alamos, NM 87545 505.665.1131 | email: tanja@lanl.gov, rickm@lanl.gov | www.lanl.gov/orgs/mpa 


\section{Center for Integrated Nanotechnologies, MPA-CINT}

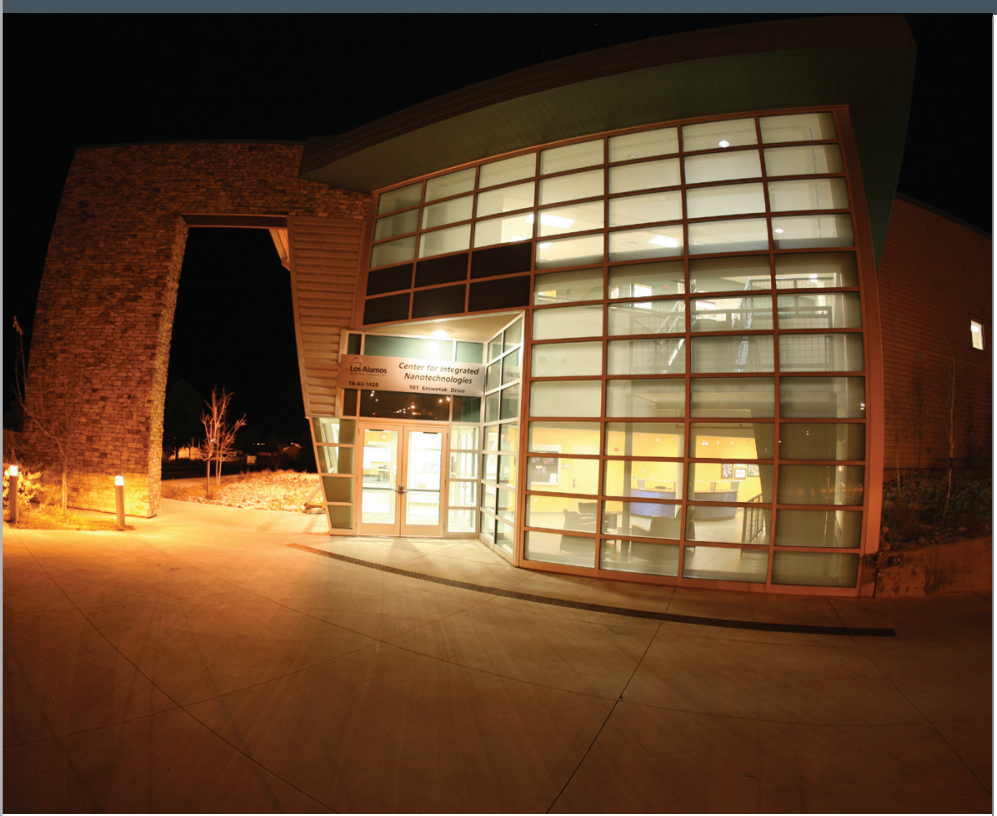

MPA-CINT is the home organization for the Center for Integrated Nanotechnologies (CINT). MPA-CINT is a diverse organization of research scientists, technicians, postdoctoral researchers, and students specializing in creating, characterizing, and understanding novel nanoscale materials and phenomena. The educational background and expertise of the staff spans biosciences, chemistry, materials sciences, physics, and engineering. MPA-CINT staff sustain a broad range of scientific activities aiding both discovery science and national security missions. In addition to staff dedicated to supporting the user facility, MPA-CINT includes the Laboratory for Ultrafast Materials and Optical Science (LUMOS). LUMOS researchers are pushing scientific boundaries in ultrafast spectroscopy, metamaterials, and terahertz wave transmission.

Key capabilities: Chemical and physical syntheses of nanoscale and nanostructured materials; biologically inspired approaches to material design; optical, electron, and force microscopies and microscopectroscopies for imaging and characterization; ultrafast science; photonics and plasmonics; metamaterials.

\section{Andreas K. Roelofs, Group Leader}

e-mail: aroelofs@lanl.gov

505.667 .2716

cint.lanl.gov

James H. Werner, Deputy Group Leader e-mail: jwerner@lanl.gov

505.667 .8842

\section{CINT \\ National User Facility}

The Center for Integrated Nanotechnologies (CINT), jointly operated by Los Alamos and Sandia national laboratories, is one of five Department of Energy Office of Science Nanoscale Science Research Centers. Through its Core Facility in Albuquerque and Gateway to Los Alamos, CINT provides open access to tools and expertise needed to develop the scientific principles governing the design, performance, and integration of nanostructured materials into the meso-, micro-, and macro-scale worlds. This differentiating focus on nanomaterials integration involves the experimental and theoretical exploration of behavior over multiple spatial and temporal length scales, the development of novel synthesis and processing approaches, and an understanding of emergent behavior and new performance regimes. Science thrusts include quantum materials systems, nanophotonics and optical nanomaterials, in situ characterization and nanomechanics, and soft biological and composite nanomaterials. CINT attracts around 450 users each year from academic, governmental, and industrial institutions based around the United States and the world.

Key capabilities: Expertise and advanced capabilities are available in nanoscale electronics and mechanics; theory, simulation, and modeling; soft, biological, and composite nanomaterials; and nanophotonics and optical nanomaterials. This comprehensive portfolio of capabilities is complemented by CINT Discovery Platforms ${ }^{\mathrm{TM}}$, which are customized microfabricated structures and devices used for nanoscience research.

\section{Andreas K. Roelofs, CINT Director}

e-mail: aroelofs@lanl.gov

505.667 .2716

cint.lanl.gov 


\section{Condensed Matter and Magnet Science, MPA-CMMS}

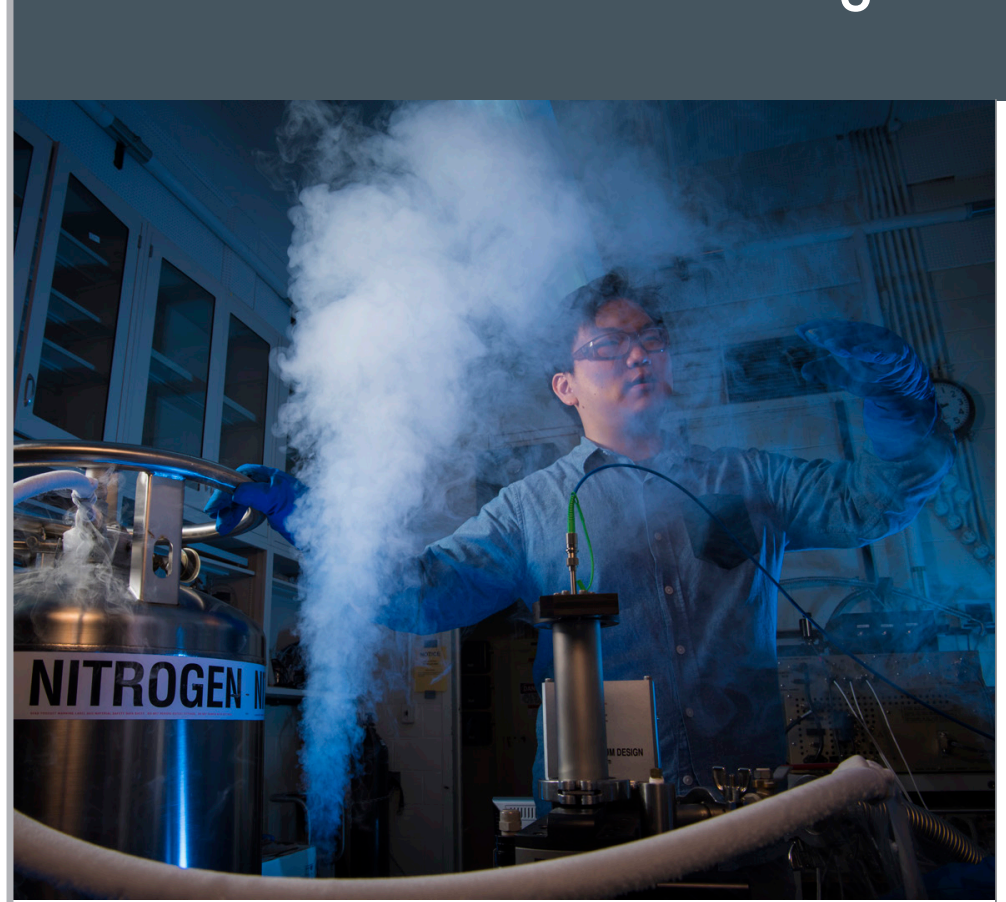

The Condensed Matter and Magnet Science group (MPA-CMMS) is comprised of research scientists, technicians, postdoctoral researchers, and students specializing in experimental physics research, with a strong emphasis on fundamental condensed matter physics and complementary thrusts in correlated electron materials, high magnetic field science and technology, actinide materials, vortex physics, energy research, and non-linear dynamics. MPA-CMMS is also home to the National High Magnetic Field Laboratory-Pulsed Field Facility, a National Science Foundation-funded pulsed magnetic field user facility.

Key capabilities: Materials synthesis; electronic transport, magnetism, and thermodynamic characterization at extreme conditions of temperature, pressure, and magnetic field; multi-shot and single-shot pulsed magnetic fields; very low temperature physics; solid-state nuclear magnetic resonance; photoelectron spectroscopy; optical spectroscopy; low temperature scanned probes; neutron scattering and diffraction; thermoacoustics; fluid dynamics; $3 \mathrm{D}$ visualization; resonant ultrasound spectroscopy.

Michael F. Hundley, Group Leader e-mail: hundley@lanl.gov

505.667.4129

www.lanl.gov/org/padste/adeps/materials-physics-applications/ condensed-matter-magnet-science/index.php

National High Magnetic Field Laboratory-Pulsed Field Facility

The National High Magnetic Field Laboratory-Pulsed Field Facility (NHMFL-PFF) user program is where the world's highest nondestructive pulsed magnetic fields are generated for qualified users of the National Science Foundation user facility. In collaboration with Florida State University and the University of Florida, Los Alamos National Laboratory hosts the pulsed magnetic field component of this national user program. The staff of the NHMFL-PFF is comprised of a dozen PhD scientists who are leaders in experimental condensed matter physics in high magnetic field, technicians with specialties in pulsed high voltage/high current capacitive and rotating electrical machinery, postdoctoral researchers, and students. About 150 users conduct experiments at the facility each year.

Key capabilities: The 100-T multi-shot magnet (100TMS) system is the centerpiece of the NHMFL-PFF capability. The 100TMS is a unique-in-the-world magnet system as it can deliver the highest magnetic fields on a millisecond timescale in a nondestructive mode. Also at the NHMFL-PFF is the $>200-T$ single turn magnet system, which is available for users, as well as the world-unique 60-T controlled waveform magnet. A 4-MJ capacitor bank is fully multiplexed for user experiments where five distinct 65-T pulsed magnets are made available for users. Measurements are routinely conducted from 0.35-350 K. Sample diagnostics include high-field optical spectroscopy, magnetic susceptibility/magnetization, magnetotransport, heat capacity to $60 \mathrm{~T}$, nanometer resolution dilatometry to $100 \mathrm{~T}$, and contactless conductivity and optical transmission to fields beyond $200 \mathrm{~T}$. Our world-record-setting magnet solenoids are designed and fabricated in-house with state-of-the-art materials and fabrication methods.

\section{Marcelo Jaime, Acting NHMFL-PFF Director} e-mail: mjaime@lanl.gov

505.667 .7625

nationalmaglab.org/user-facilities/pulsed-field-facility

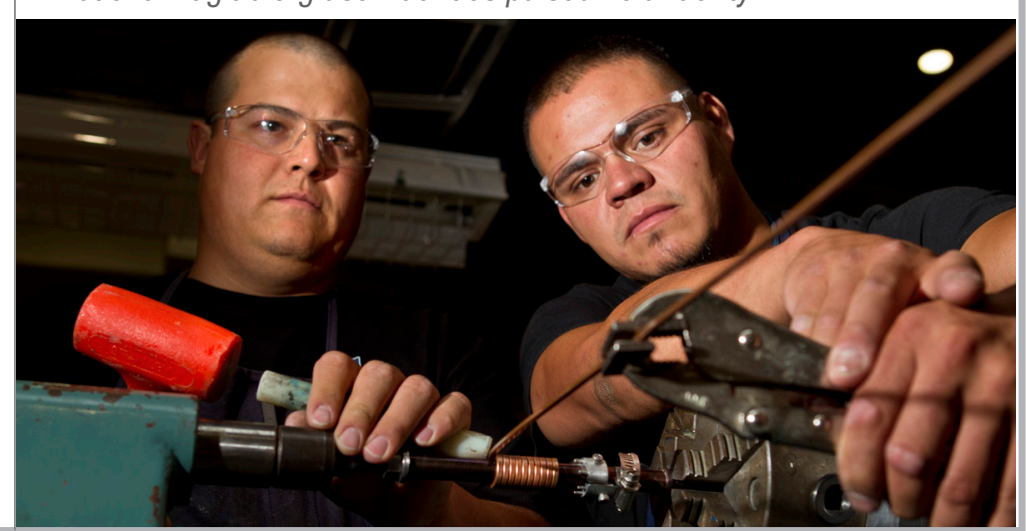




\section{MPA-11, Materials Synthesis and Integrated Devices}

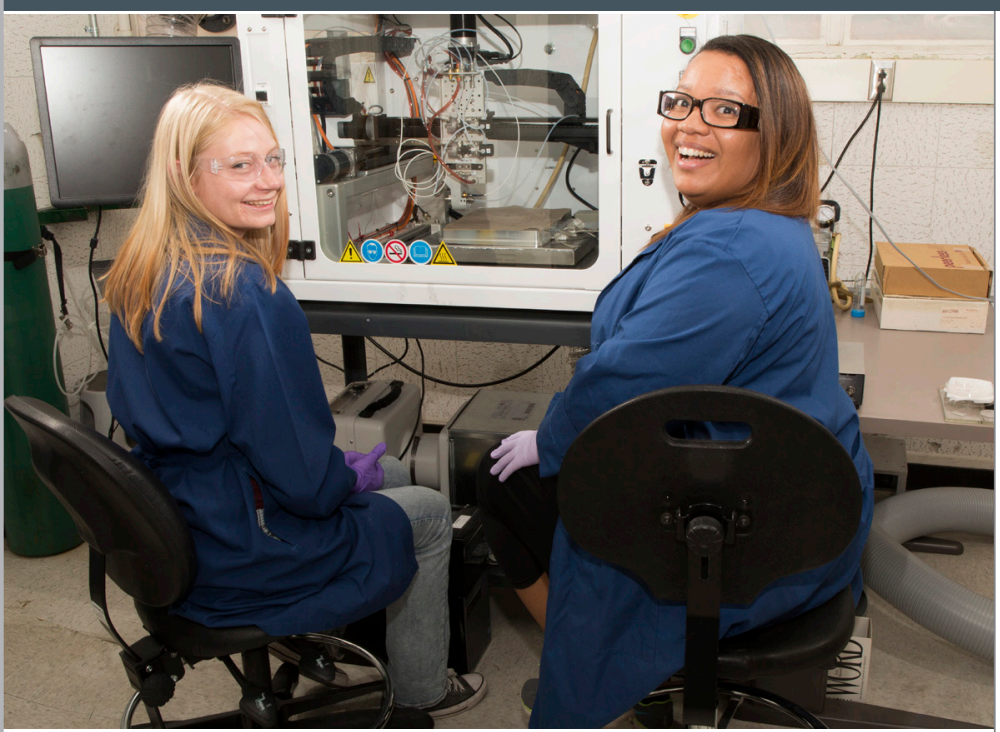

MPA-11, the Materials Synthesis and Integrated Devices group, conducts basic and applied research in areas related to applied energy programs and a variety of problems relevant to the weapons program. Our goal is to provide innovative and creative solutions needed to solve technical challenges across the Laboratory's missions, including energy security, stockpile stewardship, and global threat reduction.

Our core areas of research are focused on polymer electrolyte fuel cells for transportation and portable power; acoustic sensor technologies for enhanced fossil energy utilization; weapons aging as well as weapons safety; synthesis of actinide materials to understand bonding and reactivity; polymer membranes for greenhouse gas separations and carbon sequestration; nanomaterials synthesis and characterization for optimizing catalysis and light-to-energy conversion processes; and electrochemical-based gas/vapor sensors.

Our research forms a basis for new developments in device technology and practical application of materials. We have significant expertise in the design and incorporation of new materials into devices for a variety of applications driven by our missions. Much of our work also involves interactions with industrial sponsors and partners.
Key capabilities: Electrochemistry and electrocatalysis; fuel cell modeling, design, fabrication, and testing; design and synthesis of materials for hydrogen storage; polymer synthesis of membranes for fuel cell applications; acoustic interrogation of materials and acoustic manipulation of particles; materials development in support of stockpile stewardship and stockpile safety; molecular synthesis and actinide chemistry; advanced polymer membrane deposition and characterization for gas separations; chemical vapor deposition of two-dimensional materials, such as graphene oxide and transition metal dichalcogenides; innovative gas sensor development; proof-ofconcept device development.

\section{Andrew M. Dattelbaum, Group Leader} e-mail: amdattel@lanl.gov 505.665 .0142

\section{George S. Goff, Deputy Group Leader} e-mail: georgeg@lanl.gov 505.664.0337

\section{Jeffrey O. Willis, Deputy Group Leader} e-mail: jwillis@lanl.gov

505.667.2144

www.lanl.gov/org/padste/adeps/materials-physics-applications/ materials-synthesis-integrated-devices/index.php

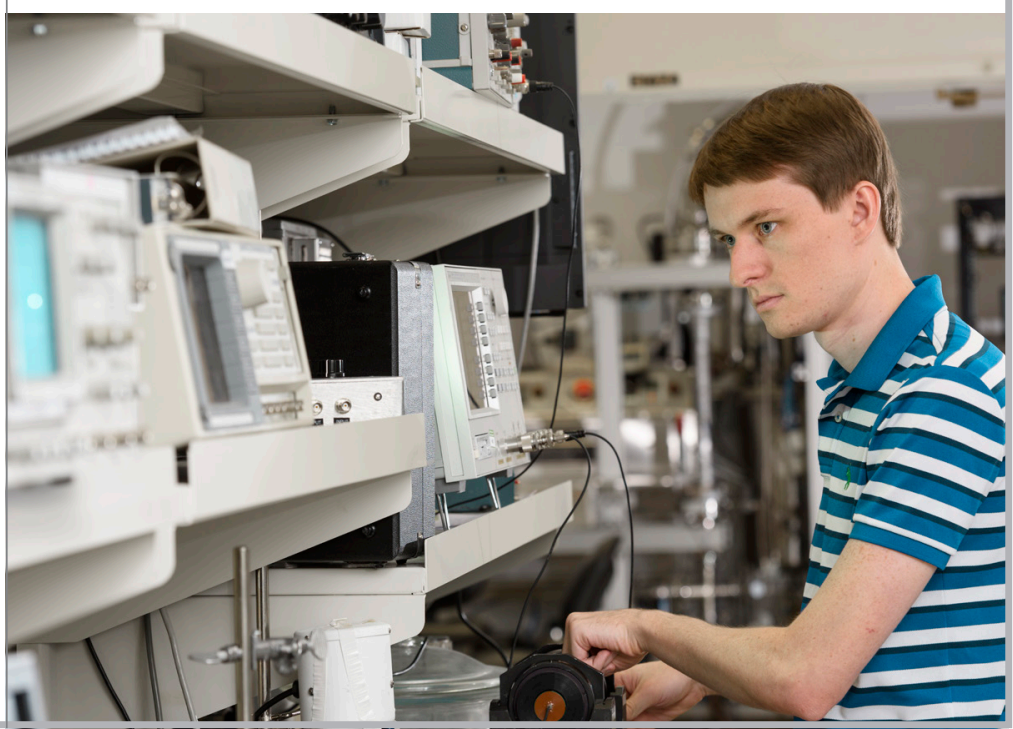

